\section{Doenças respiratórias agudas: um estudo das desigualdades em saúde}

\author{
Acute respiratory diseases: a study on health \\ inequalities
}

\author{
${ }^{1}$ Escola de Enfermagem, \\ Universidade de São Paulo, \\ São Paulo, Brasil. \\ 2 Faculdade de Saúde \\ Pública, Universidade de São \\ Paulo, São Paulo, Brasil. \\ ${ }^{3}$ Faculdade de Medicina do \\ $A B C$, Santo André, Brasil. \\ Correspondência \\ M. Akerman \\ Faculdade de Medicina do \\ $A B C$. \\ Rua Dona Brígida 232, \\ casa 32, São Paulo, SP \\ 04111-080, Brasil. \\ akermanm.ops@terra.com.br
}

\section{Abstract}

This article discusses health inequalities based on acute childhood respiratory diseases in the coverage area of a health center in the city of São Paulo, Brazil, to help plan local health promotion activities. The work was based on ecological studies using the geographic area as the unit of analysis, allowing a comparison of health and socioeconomic indicators based on census data. Indicators were constructed for "social inclusion" and "housing quality", generating the "potential exposure index", which reflects the respiratory disease risk conditions. Statistical treatment included grouping according to the cluster technique. Four homogenous social groups were identified in terms of risk conditions for acute respiratory diseases. Groups III and IV, with the worst socioeconomic conditions, showed important differences in comparison to groups I and II. The differences in mortality from pneumonia suggest important health inequalities. The results allow the geographic localization of the highest and lowest concentration of needs in terms of living conditions and the comparison of census tracts for recognizing distinct needs, thus supporting proposals for inter-sector collaboration.

Respiratory Tract Diseases; Child Welfare; Health Inequalities
Anna M. Chiesa 1

Marcia F. Westphal 2

Marco Akerman 3

\section{Introdução}

As doenças respiratórias na infância (no presente estudo a infância foi considerada como o período até cinco anos de idade) têm constituído a cada dia motivo de preocupação para os profissionais de saúde, dada a sua elevada morbidade, observada em termos mundiais, bem como a alta mortalidade que incide especialmente nos países do terceiro mundo. Segundo dados divulgados pela Organização Mundial da Saúde (OMS), cerca de 13 milhões de crianças menores de cinco anos morrem anualmente no mundo por doenças do aparelho respiratório e $95 \%$ delas ocorrem nos países em desenvolvimento 1,2,3.

No Brasil, as doenças respiratórias são responsáveis por aproximadamente $10 \%$ das mortes entre os menores de um ano, a segunda causa de óbito na população de zero a um ano de idade e a primeira causa entre as crianças de um a quatro anos 2 . Predominam no primeiro grupo as pneumonias de origem bacteriana 3,4 .

Atualmente, em termos nacionais, os problemas respiratórios constituem a segunda causa dos óbitos das regiões Sul e Sudeste e a terceira causa nas demais regiões. Em São Paulo, a mortalidade pelas infecções respiratórias agudas (resfriados, otites, sinusites, amidalites, problemas das vias inferiores, epiglotites, bronquites e pneumonias) também é significativa, ocupando o terceiro lugar na população infantil, apesar de ser o estado que conta com os melhores índices 
de sobrevivência para a população de zero até seis anos de idade, segundo estudo realizado pelo Instituto Brasileiro de Geografia e Estatística (IBGE) e pelo Fundo das Nações Unidas para a Infância (UNICEF) 5 .

Alguns estudos indicam uma relação entre condições de vida e incidência de doenças respiratórias na população infantil 6,7,8,9,10. Elucidar o papel das determinações sociais no cenário epidemiológico das doenças respiratórias na infância pode ser de grande utilidade para subsidiar políticas mais eficazes para o controle dessas doenças.

Dentro da política de descentralização do Sistema Único de Saúde (SUS) destaca-se o nível local de atenção à saúde como o espaço privilegiado para a identificação das necessidades de saúde. De acordo com Paganini \& Capote Mir 11, no nível local se fortalece a participação social, a ação intersetorial, a descentralização das decisões, a utilização de métodos de planejamento ascendente e a formulação de políticas voltadas para a realidade local.

O presente estudo tem como base o conceito de eqüidade desenvolvido na década de 9012,13 , centrado no desafio de identificar diferentes problemas de saúde para que possam ser desencadeadas intervenções diferenciadas. O enfoque se centra nas possibilidades de controle das doenças respiratórias na infância em um dado espaço geográfico que se constitui como uma área de abrangência de uma unidade básica de saúde na cidade de São Paulo, revelando um exercício de articulação de dois princípios fundamentais do SUS, quais sejam a universalidade e a eqüidade.

Neste estudo são analisadas as desigualdades em saúde, valendo-se das doenças respiratórias agudas na infância, da população residente na área de abrangência do Centro de Saúde Escola Samuel B. Pessoa, na região do Butantã, na cidade de São Paulo; tendo em vista subsidiar o planejamento das ações de promoção da saúde em nível local. Para tanto contempla: (1) a descrição da metodologia para caracterizar os potenciais de exposição às doenças respiratórias na infância, do ponto de vista de distintos atores sociais locais; (2) a distribuição desses potenciais de exposição nas 49 unidades censitárias da região, evidenciando os diferenciais de mortes por doenças respiratórias e por pneumonias nos menores de cinco anos entre grupos homogêneos construídos pelas condições sócio-econômicas; (3) algumas recomendações no campo da promoção da saúde para o enfrentamento das doenças respiratórias na infância.

\section{Metodologia}

\section{Cenário do estudo}

O Centro de Saúde Escola Samuel B. Pessoa foi escolhido como unidade básica de saúde de referência porque tem assumido um papel de vanguarda pela implementação de alternativas inovadoras no campo da assistência, ensino e pesquisa do tema. Trata-se de um centro de saúde resultante de um convênio entre a Secretaria Estadual de Saúde de São Paulo e a Faculdade de Medicina da Universidade de São Paulo 14.

A população estudada foi aquela que residia na área de abrangência do Centro de Saúde Escola Samuel B. Pessoa. Dada a alta incidência dos agravos respiratórios no perfil de morbidade da população infantil, o enfoque da presente investigação voltou-se para a totalidade das famílias residentes na área delimitada, na perspectiva de conhecer as iniqüidades presentes na população em tela, para obter subsídios para a implantação de propostas de intervenção.

\section{Marco teórico metodológico}

No delineamento deste estudo foram fundamentais as proposições de Santos 15 e Unglert 16. Para esses autores, o território assume uma dimensão diferenciada na formulação de políticas públicas, dentre as quais as políticas de saúde.

Para Santos 15, a globalização tem sido um processo prejudicial à dimensão da vida, pois não respeita as condições históricas e reais de todos os envolvidos. Ao invés de proporcionar a união entre as diferentes culturas e valores existentes, promove a apropriação universalizada do trabalho fragmentado, desvalorizando as especificidades locais. Um dos núcleos de resistência às conseqüências destrutivas desse processo é o espaço local que pode contribuir com o fortalecimento dos segmentos sociais que detêm menor poder, pelo fato de constituir cenário do "acontecer solidário". Conceber o território como espaço-local e como pólo distinto da globalização, leva à descoberta de aspectos relevantes na reprodução social dos atores nele envolvidos.

No trabalho de Unglert 16, destacam-se as contribuições da geografia humana, no embasamento desse conceito e na organização do processo de "oficinas de territorialização" como forma de operar intervenções em saúde no âmbito do SUS.

Os espaços que constituem os locais de moradia escondem imensas desigualdades, sobretudo nas grandes metrópoles, onde se evidenciam grandes extensões territoriais carentes de serviços. 
Não se trata de uma recuperação idealista do conceito de comunidade traçando uma perspectiva utópica no sentido de inatingível, de que seria uma solução para todos os males atuais. Tampouco, trata-se de uma nova aproximação junto ao espaço comunitário nos moldes do que ocorreu na década de 50 sob a política de "desenvolvimento da comunidade", consoante a descrição de Ammann 17, através da qual estabelecia-se uma relação de dominação cultural dos países centrais sobre os povos economicamente dependentes.

O desafio atual para subsidiar as ações comunitárias seria a aproximação com vistas ao desenvolvimento de trabalhos centrados na perspectiva da valorização do local, explorando as potencialidades da vivência solidária como base para o fortalecimento da identidade da cidadania.

Na área da saúde, vários autores 16,18 relatam a utilização desse quadro conceitual como elemento fundamental para a construção de uma nova abordagem dos serviços em relação ao território que ocupam.

Paim 19 descreve diferentes abordagens que têm sido utilizadas na compreensão da relação entre a estrutura social e as condições de saúde, salientando ainda, a importância do reconhecimento da intervenção do Estado, por intermédio das políticas públicas, no atendimento das necessidades sociais dos diferentes grupos.

Paim destaca ainda a propriedade da base territorial para a realização de investigações epidemiológicas voltadas para as condições de vida e inserção espacial dos grupos humanos, utilizando o território não somente na perspectiva física ou natural, mas como processo histórico e social, podendo constituir uma alternativa teórico-metodológica para a análise das necessidades e desigualdades sociais.

Para fundamentar ainda mais o presente estudo, recuperou-se a experiência de outros trabalhos que evidenciaram as iniqüidades decorrentes da inserção desigual dos indivíduos na sociedade. Neste sentido, destaca-se o trabalho coordenado por Sposati 20, no qual foram utilizados indicadores compostos para evidenciar as desigualdades sociais existentes na cidade de São Paulo.

O presente estudo também contemplou o delineamento metodológico dos estudos ecológicos que utilizam a área geográfica como unidade de análise, permitindo a comparação de indicadores de saúde e sócio-econômicos mediante dados obtidos no Censo Demográfico de 199121.

Para Borrell 22, tais estudos oferecem um produto diverso daqueles que se fundamentam nos dados relativos aos indivíduos, visto que medem outro fenômeno relativo ao ambiente social onde os indivíduos vivem, além do fato de considerarem as causas sociais das doenças, podendo auxiliar na formulação de políticas voltadas para os problemas sócio-sanitários. Castairs (1981, apud Borrel 22), que trabalhou com variáveis censitárias e Castairs \& Morris (1988, apud Borrel 22), constituem exemplos de estudos ecológicos baseados nos índices de privação, de grande contribuição para a identificação de problemas e formulação de políticas específicas.

Castellanos 23 também destaca a contribuição de estudos que utilizam a dimensão ecológica para a saúde pública, particularmente, no caso da formulação de ações específicas para os diferentes perfis de saúde dos diferentes grupos sociais e para produzir impacto sobre as iniqüidades sociais em saúde.

\section{Etapas da investigação}

\section{- A utilização de dados quantitativos}

Tendo em vista a caracterização da base territorial que conforma a área de abrangência do centro de saúde, primeiramente foram identificados os setores censitários componentes dela.

O IBGE forneceu dados sobre o conjunto de 49 setores censitários componentes da área de abrangência em estudo disponibilizando um conjunto de 294 variáveis referentes aos domicílios particulares permanentes pertencentes à área.

Para atender aos objetivos descritos anteriormente, foram selecionadas algumas variáveis que permitissem a caracterização da inserção social da população residente na área. Com isso, buscou-se identificar situações de exposição a diferentes gradientes de condições favoráveis à vida e de risco para o adoecimento por agravos respiratórios, tomando como foco o coletivo como um todo, incorporando o conceito de eqüidade em relação à saúde, distinguindo-se assim do conceito muitas vezes utilizado relativo ao consumo dos serviços de saúde. O ponto de origem deste trabalho foi a identificação das iniqüidades existentes para poder contemplar a eqüidade no processo de formulação da intervenção no âmbito da promoção à saúde.

Tal caracterização foi baseada na construção de indicadores compostos que constituem "medida que associa diferentes variáveis sócioeconômicas e de ambiente num indicador sintético para analisar as características de grupos populacionais vivendo em determinadas áreas geográficas” 24 (p. 374).

Dentre as variáveis disponíveis no banco de dados fornecido pelo IBGE, foi identificado, primeiramente, um conjunto que pudesse retratar 
os aspectos de inserção social dos sujeitos e outro conjunto mais voltado para as condições do domicílio, dada a importância que as condições têm no desencadeamento dos agravos respiratórios. Os conjuntos de variáveis foram denominados "indicadores de inserção social" (IIS) e "indicadores de qualidade do domicílio" (IQD), e, ao final, compuseram o "índice de potencial de exposição” às condições de risco ambiental para o adoecimento por agravos respiratórios.

a) Indicador de inserção social (IIS): renda do chefe do domicílio, escolaridade do chefe, situação de ocupação do domicílio. Assim, o indicador incluiu a escolaridade do chefe, a renda per capita, que foi calculada, e a situação de ocupação do domicílio por entender que esta última seria uma possibilidade de retratar uma condição de maior ou menor estabilidade no vínculo com a moradia e, possivelmente, a fixação no território, permanente ou temporária.

b) Indicador de qualidade do domicílio (IQD): acesso à água, condições sanitárias, aglomeração, coleta de lixo, tipo de construção. Com relação ao IQD, foram selecionadas cinco variáveis para retratar as condições de conforto em termos de exposição da criança às friagens ou a outro fator deletério à sua saúde, que pudesse atuar como um facilitador do adoecimento por infecções respiratórias agudas. Incluiu as variáveis: acesso à água; condições sanitárias; aglomeração (calculada pelo número médio de dormitórios e número de pessoas dos domicílios); coleta de lixo e tipo de construção.

Os aspectos sociais associados aos mesmos descritos na literatura destacam: a baixa escolaridade dos pais; o maior número de pessoas no domicílio; a baixa idade materna; a permanência em período integral em creches ou instituições congêneres; precárias condições sócio-econômicas e habitacionais da família 7,25,26,27.

Em função disso, foram identificadas no banco de dados disponíveis as variáveis que correspondiam aos riscos já conhecidos, utilizados para a construção dos indicadores compostos que formaram a base instrumental para a comparação dos setores censitários com intuito de identificar as desigualdades sociais potencialmente geradoras de iniqüidades para a saúde.

\section{- A participação de outros atores sociais na construção do instrumental metodológico}

A seleção das variáveis levou em conta tanto condições de risco descritas na literatura, relativos ao adoecimento e à morte por doenças em crianças, como também resultados de um processo de discussão por meio da estratégia de grupos focais junto a lideranças locais para a definição conjunta do peso de cada variável na composição do índice em tela. O procedimento utilizado seguiu as recomendações de Akerman et al. 24 , que postulam como de fundamental importância que as variáveis sejam definidas valendo-se de processos participativos, a fim de incorporar as percepções de outros sujeitos, além dos técnicos.

De acordo com o referencial teórico da promoção à saúde, que preconiza a aproximação entre técnicos e população mediante processos de discussão conjunta dos problemas e encaminhamentos possíveis para sua superação, e, tendo em vista a finalidade deste estudo de não reproduzir uma prática de diagnóstico da situação apenas unilateralmente, por parte dos técnicos de um setor de prática de políticas públicas, buscou-se contemplar este aspecto por intermédio da discussão sistematizada junto aos setores representativos da população local, utilizando-se a técnica de grupo focal. Foram realizados dois encontros com representantes da comunidade para apresentar esta proposta de conjunto de variáveis e incorporar as sugestões. As discussões foram realizadas em duas datas diferentes, reunindo representantes do Conselho Tutelar da Criança e do Adolescente do Butantã, técnicos do Centro de Educação Ambiental, representantes do Parque da Previdência e membros da Pastoral da Saúde e da Criança, envolvendo um total de 21 pessoas, sendo 8 participantes no primeiro grupo e 13 no segundo grupo.

As discussões com a população confirmaram a proposta inicial no tocante à indicação das variáveis e tiveram como resultado a ponderação delas para a construção dos indicadores, bem como o peso dos indicadores na construção do índice. Ou seja, diante dos dados disponibilizados pelo IBGE, houve consenso em utilizar as variáveis propostas, não tendo sido acrescentada nem retirada nenhuma delas. $\mathrm{O}$ resultado final dos grupos focais foi o esquema de hierarquização das variáveis. Com relação ao IIS, foi sugerido que o peso maior recaísse sobre a escolaridade; com relação ao IQD foi indicado que o peso maior deveria ser dado ao número de pessoas por dormitório, denominado no estudo por condições de aglomeração (peso 3), seguido de condições sanitárias e tipo de construção (peso 2) e, finalmente, acesso à água e à coleta de lixo (peso 1).

Quanto à conformação do "índice de potencial de exposição" às condições de risco ambiental para os agravos respiratórios, o que representa a síntese dos dois indicadores, foi sugerido que o IIS tivesse peso 2 sobre o IQD. 


\section{- Tratamento estatístico dos dados}

Os dados dos setores censitários receberam tratamento estatístico, tendo sido calculada a média ponderada de cada setor em cada variável selecionada, que representa a situação típica da mesma em cada setor. Em seguida, as variáveis componentes dos indicadores receberam os diferentes pesos para o cálculo. Chegou-se, então, ao seguinte esquema para compor os indicadores.

Indicador de inserção social (IIS) $=$ renda $+(2 x$ escolaridade) + ocupação do domicílio/4

Indicador de qualidade do domicílio (IQD) = água + 2 x condições sanitárias $)+(3 \mathrm{x}$ aglomeração) + ( 2 x tipo de construção $)+$ lixo/9

O tratamento estatístico realizado envolveu o cálculo da média ponderada de cada variável por setor censitário. A condição que predominava no setor era classificada segundo critérios apontados em outros estudos, sobretudo no trabalho de mensuração da pobreza da cidade de São Paulo que foi realizado pela Fundação Sistema Estadual de Análise de Dados (Fundação SEADE) 28 , e era atribuída uma nota representativa da mesma para fins de comparação entre os setores. Os cálculos foram efetuados utilizandose o programa Excel (Microsoft Corp., Estados Unidos)

A melhor condição possível recebeu o valor positivo 1 e a pior condição recebeu o valor negativo de -1 , considerando-se os percentuais existentes em cada fração das variáveis, de forma análoga ao cálculo da média ponderada em termos absolutos.

Com base nos conjuntos de indicadores, buscou-se estabelecer a relação entre ambos para se chegar ao índice de exposição ambiental para os agravos respiratórios pela expressão abaixo.

Índice de potencial de exposição $=2 \times$ IIS + $\mathrm{IQD} / 3$

Os setores censitários foram comparados e chegou-se a um ranking deles pelo estabelecimento dos diferentes potenciais de adoecimento por doenças respiratórias agudas na infância.

\section{Resultados e discussão}

\section{Caracterização do território}

Pela delimitação geográfica dos setores fornecida pelo IBGE, utilizando-se o programa MapInfo (MapInfo Corp., Nova York, Estados Unidos), chegou-se ao mapa da área de abrangência do Centro de Saúde Escola Samuel B. Pessoa (Figura 1).

A área apresentada na Figura 1 apresentava em 1991, segundo o último censo disponível, um total de 47.511 habitantes abrigando 12.295 domicílios, sendo 4.170 crianças menores de cinco anos.

A Tabela 1 apresenta os valores numéricos representativos das variáveis componentes relativas à "inserção social” e a Tabela 2 apresenta os valores numéricos representativos das condições que expressam a "qualidade dos domicílios” em cada setor censitário. Os referidos valores numéricos foram utilizados na expressão matemática para se chegar aos indicadores.

Com isso, pode-se calcular os valores de IIS e IDQ de cada setor censitário para posteriormente efetuar o cálculo que expressou a situação dos setores em termos do "índice potencial de exposição” para as condições de risco para os agravos respiratórios.

Em seguida, os setores foram ordenados de forma decrescente, segundo o valor final comparativo, chegando-se a um ranking dos diferentes potenciais de exposição às condições de risco para o adoecimento por doenças respiratórias agudas, apresentados na Tabela 3.

\section{O território a partir das desigualdades nas condições de vida}

Tomando-se por base o ranking citado, foi utilizada a técnica de análise de agrupamentos cluster do pacote estatístico MiniTab (MiniTab Brasil, Belo Horizonte, Brasil) para a identificação de quatro grupos homogêneos dos setores censitários, tendo em vista a perspectiva de reconhecimento das diferentes necessidades e a proposição de intervenções junto a tais grupos. Na coluna da direita da Tabela 3, pode-se identificar a composição de quatro grupos homogêneos em termos do potencial de exposição às condições de risco para as doenças respiratórias agudas: o grupo I reúne as melhores condições da área estudada; o grupo II reflete uma situação intermediária/boa, na medida em que agrupa somente setores que obtiveram valores positivos e acima do valor médio de 0,5 ; o grupo homogêneo III apresenta uma situação regular/ ruim, na medida em que congrega setores com valores positivos baixos e negativos; e o grupo IV que reúne as piores condições para a população residente.

Com base na territorialização em saúde, foram localizados os diversos grupos homogêneos, utilizando-se o programa MapInfo. A Figura 2 apresenta a área de abrangência do centro de saúde, destacando-se os grupos homogêneos em termos do potencial de exposição às condições de risco para as doenças respiratórias agudas. 


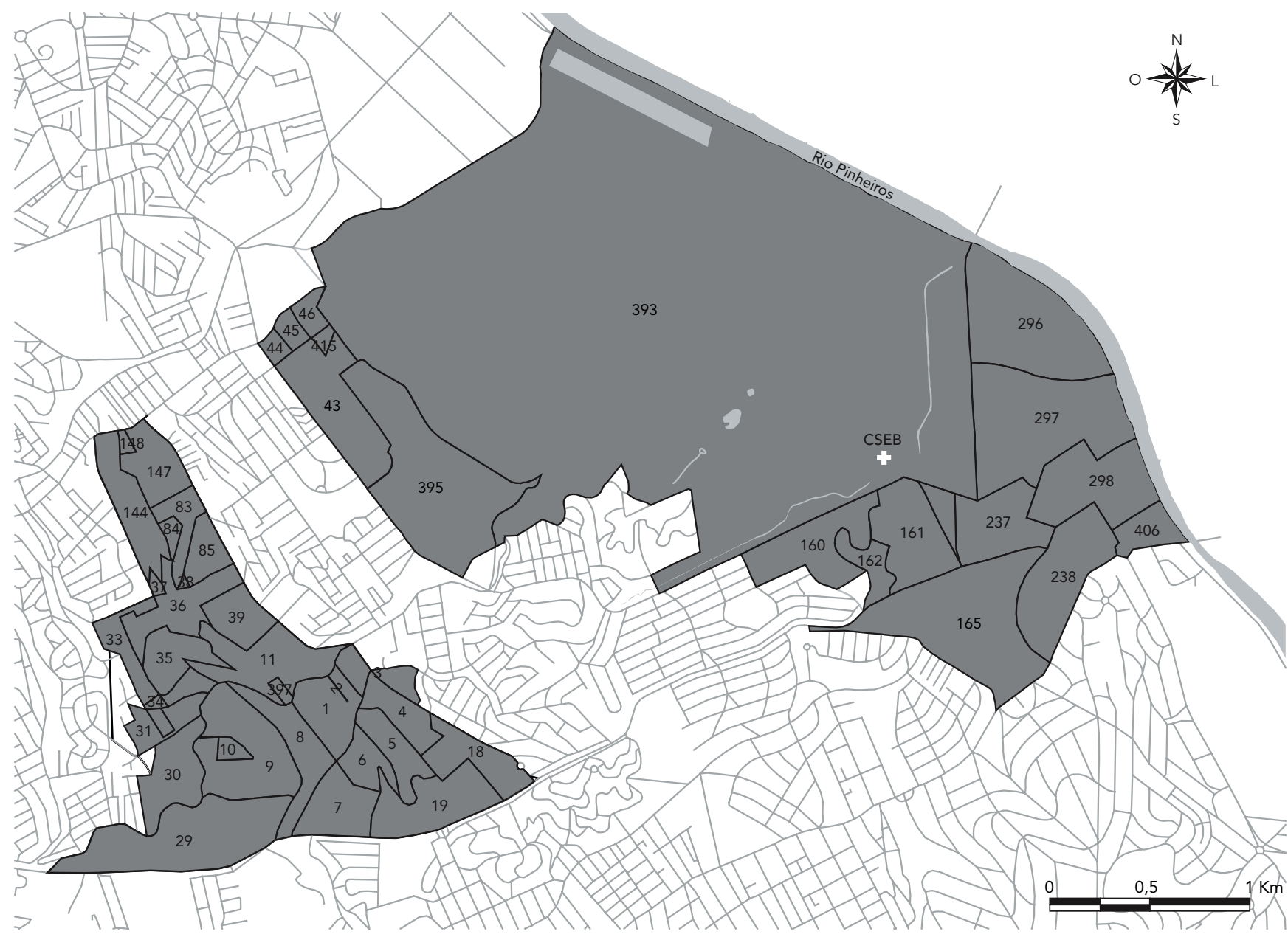

A Figura 2 caracteriza as desigualdades sociais identificadas na microárea de estudo que se configura enquanto um espaço para atuação local na área da saúde. Configura-se também como um espaço de ação intersetorial ao se considerar o leque de necessidades da população a ser contemplado pelos equipamentos públicos que atuam na mesma área. Nesse sentido, acredita-se que este reconhecimento, com base nas condições de vida, pode contribuir para uma aproximação intersetorial profícua com o objetivo do fortalecimento de uma nova postura ao assumir a tarefa de formulação e gestão de políticas públicas.

Esse reconhecimento da realidade local é importante para a reorientação das ações propos- tas, tendo em vista o referencial teórico adotado no presente estudo. Um aspecto a ser destacado inicialmente com base nos grupos homogêneos identificados é a própria composição em termos de concentração da população total e da concentração de crianças menores de cinco anos, conforme pode ser verificado nas Figuras 3 e 4, respectivamente.

Vale destacar a importância que assumem os grupos homogêneos III e IV com relação à concentração de crianças menores de cinco anos, reforçando a necessidade de implementação de ações diferenciadas com base na realidade local.

Por intermédio desses dados foi possível conhecer a ocorrência dos óbitos de menores de 
Tabela 1

Valores obtidos pelos setores censitários nas variáveis relativas ao indicador de inserção social (ISS). Butantã, São Paulo, Brasil, 1998.

\begin{tabular}{|c|c|c|c|}
\hline Setor censitário & $\begin{array}{c}\text { Índice de } \\
\text { escolaridade }\end{array}$ & $\begin{array}{l}\text { Índice de renda } \\
\text { per capita }\end{array}$ & $\begin{array}{l}\text { Índice de condição de } \\
\text { ocupação do domicílio }\end{array}$ \\
\hline 1 & 0,8502 & 0,6781 & 0,9397 \\
\hline 2 & 0,9125 & 0,8372 & 0,9317 \\
\hline 3 & 0,6389 & $-0,2042$ & 1,0000 \\
\hline 4 & 0,8057 & 0,6658 & 0,9236 \\
\hline 5 & 0,6817 & $-0,1722$ & 0,9193 \\
\hline 6 & 0,6830 & 0,6748 & 0,9014 \\
\hline 7 & 0,6332 & $-0,2056$ & 0,9016 \\
\hline 8 & $-0,3275$ & $-0,2658$ & 0,8851 \\
\hline 9 & 0,6434 & $-0,2385$ & 0,8798 \\
\hline 10 & $-0,8746$ & $-0,8028$ & $-0,9980$ \\
\hline 11 & $-0,4184$ & $-0,3382$ & $-0,8286$ \\
\hline 18 & 0,8014 & 0,6033 & 0,9403 \\
\hline 19 & 0,6077 & $-0,2261$ & 0,8900 \\
\hline 29 & $-0,3199$ & $-0,2547$ & 0,8495 \\
\hline 30 & 0,7768 & $-0,1692$ & 0,8609 \\
\hline 31 & $-0,4601$ & $-0,4658$ & $-0,8456$ \\
\hline 32 & $-0,9637$ & $-0,8621$ & $-1,0000$ \\
\hline 33 & $-0,4621$ & $-0,5587$ & $-0,8331$ \\
\hline 34 & $-1,0000$ & $-0,9643$ & $-1,0000$ \\
\hline 35 & $-0,4092$ & $-0,3673$ & $-0,8149$ \\
\hline 36 & $-0,3842$ & $-0,4351$ & $-0,8233$ \\
\hline 37 & $-0,5877$ & $-0,7728$ & $-1,0000$ \\
\hline 38 & $-0,8398$ & $-0,7960$ & $-0,9862$ \\
\hline 39 & $-0,3817$ & $-0,3143$ & 0,8938 \\
\hline 43 & 0,6884 & $-0,2003$ & 0,9607 \\
\hline 44 & $-0,7060$ & $-0,7003$ & $-0,9958$ \\
\hline 45 & $-0,6892$ & $-0,7015$ & $-0,9933$ \\
\hline 46 & $-0,6788$ & $-0,5328$ & $-0,9851$ \\
\hline 83 & $-0,3563$ & $-0,3591$ & 0,8689 \\
\hline 84 & $-0,8150$ & $-0,8637$ & $-0,9964$ \\
\hline 85 & 0,6628 & 0,6011 & 0,8888 \\
\hline 144 & $-0,4293$ & $-0,4309$ & $-0,8507$ \\
\hline 147 & $-0,3387$ & $-0,2884$ & 0,8872 \\
\hline 148 & $-0,8164$ & $-1,0000$ & $-0,9668$ \\
\hline 160 & $-0,3192$ & $-0,2864$ & $-0,8264$ \\
\hline 161 & 0,7411 & $-0,1996$ & 0,8912 \\
\hline 162 & $-0,3415$ & $-0,3726$ & $-0,8406$ \\
\hline 165 & 0,8114 & 0,9591 & 0,8659 \\
\hline 236 & 0,9367 & 0,8686 & 0,8774 \\
\hline 237 & 0,8460 & 1,0000 & 0,8442 \\
\hline 238 & 0,7185 & 0,6500 & 0,8662 \\
\hline 296 & 1,0000 & 0,7898 & 0,9931 \\
\hline 297 & 0,9855 & 0,7892 & 0,9786 \\
\hline 298 & 0,8424 & 0,6906 & $-0,8339$ \\
\hline 393 & 0,7520 & 0,6062 & 0,8420 \\
\hline 395 & $-0,3908$ & $-0,3200$ & 0,8594 \\
\hline 397 & $-0,3633$ & $-0,2271$ & 0,8731 \\
\hline 406 & 0,8484 & 0,9670 & $-0,9701$ \\
\hline 415 & $-1,0000$ & $-0,6793$ & $-1,0000$ \\
\hline
\end{tabular}


Tabela 2

Valores obtidos pelos setores censitários nas variáveis relativas ao indicador de qualidade do domicílio (IQD). Butantã, São Paulo, Brasil, 1998.

\begin{tabular}{|c|c|c|c|c|c|}
\hline Setor censitário & $\begin{array}{c}\text { Índice de } \\
\text { acesso à água }\end{array}$ & $\begin{array}{c}\text { Índice de } \\
\text { condições sanitárias }\end{array}$ & $\begin{array}{c}\text { Índice de } \\
\text { aglomeração }\end{array}$ & $\begin{array}{c}\text { Índice de condições } \\
\text { de coleta de lixo }\end{array}$ & $\begin{array}{l}\text { Índice de tipo } \\
\text { de construção }\end{array}$ \\
\hline 1 & 1,0000 & 0,9862 & 0,8867 & 1,0000 & 0,9948 \\
\hline 2 & 1,0000 & 0,9994 & 0,8867 & 0,9992 & 0,9985 \\
\hline 3 & 1,0000 & 0,9961 & 0,8867 & 0,9922 & 1,0000 \\
\hline 4 & 1,0000 & 1,0000 & 0,8867 & 1,0000 & 1,0000 \\
\hline 5 & 1,0000 & 1,0000 & 0,8867 & 1,0000 & 1,0000 \\
\hline 6 & 1,0000 & 0,9911 & 0,8867 & 1,0000 & 0,9980 \\
\hline 7 & 0,9981 & 0,9944 & 0,8867 & 0,9981 & 0,9963 \\
\hline 8 & 0,9983 & 0,9701 & $-0,7500$ & 0,9983 & 0,9913 \\
\hline 9 & 0,9976 & 0,9804 & 0,6650 & 0,9903 & 0,9793 \\
\hline 10 & 0,9318 & 0,7657 & $-1,0000$ & $-0,9985$ & $-0,9589$ \\
\hline 11 & 0,9951 & 0,9538 & $-0,7500$ & 1,0000 & 1,0000 \\
\hline 18 & 1,0000 & 0,9980 & 0,8867 & 1,0000 & 1,0000 \\
\hline 19 & 1,0000 & 0,9504 & 0,8867 & 1,0000 & 1,0000 \\
\hline 29 & 0,9973 & 0,9653 & $-0,7500$ & 0,9728 & 1,0000 \\
\hline 30 & 0,9964 & 0,9740 & $-0,7500$ & 0,9964 & 1,0000 \\
\hline 31 & 1,0000 & 0,9512 & $-0,7500$ & 1,0000 & 1,0000 \\
\hline 32 & 0,9137 & $-0,7427$ & $-1,0000$ & 1,0000 & $-0,9966$ \\
\hline 33 & 0,9990 & 0,8779 & $-1,0000$ & 0,9727 & 0,9854 \\
\hline 34 & 1,0000 & 0,9333 & $-1,0000$ & 1,0000 & $-0,9966$ \\
\hline 35 & 1,0000 & 0,9057 & $-0,7500$ & 1,0000 & 0,9762 \\
\hline 36 & 1,0000 & 0,9534 & $-0,7500$ & 1,0000 & 1,0000 \\
\hline 37 & 0,9896 & $-0,7475$ & $-1,0000$ & $-0,9878$ & $-0,9843$ \\
\hline 38 & 0,9580 & 0,8969 & $-1,0000$ & $-1,0000$ & $-0,9966$ \\
\hline 39 & 1,0000 & 0,9684 & $-0,7500$ & 1,0000 & 1,0000 \\
\hline 43 & 1,0000 & 0,9873 & $-0,7500$ & 0,9969 & 0,9939 \\
\hline 44 & 0,9776 & $-1,0000$ & $-1,0000$ & $-0,9966$ & $-0,9966$ \\
\hline 45 & $-1,0000$ & $-0,9559$ & $-1,0000$ & 0,9195 & $-1,0000$ \\
\hline 46 & $-0,5955$ & 0,7810 & $-1,0000$ & 0,9725 & $-0,8977$ \\
\hline 83 & 0,9977 & 0,9966 & $-0,7500$ & 0,9627 & 1,0000 \\
\hline 84 & 0,8575 & $-0,7063$ & $-1,0000$ & $-0,8922$ & $-0,9966$ \\
\hline 85 & 1,0000 & 1,0000 & 0,8867 & 1,0000 & 1,0000 \\
\hline 144 & 0,9891 & 0,9546 & $-0,7500$ & 0,9993 & 0,9748 \\
\hline 147 & 0,9982 & 0,9925 & $-0,7500$ & 1,0000 & 0,9946 \\
\hline 148 & $-0,8809$ & $-0,7370$ & $-1,0000$ & 1,0000 & $-0,9966$ \\
\hline 160 & 0,9946 & 0,9790 & $-0,7500$ & 1,0000 & 0,9729 \\
\hline 161 & 0,9971 & 0,9849 & 0,6650 & 1,0000 & 1,0000 \\
\hline 162 & 1,0000 & 0,9124 & $-0,7500$ & 1,0000 & 1,0000 \\
\hline 165 & 0,9982 & 0,9707 & 0,8867 & 1,0000 & 1,0000 \\
\hline 236 & 0,9912 & 0,9992 & 0,8867 & 0,9989 & 0,9956 \\
\hline 237 & 0,9982 & 0,9877 & 0,6650 & $-0,9233$ & 1,0000 \\
\hline 238 & 0,9972 & 0,9808 & 0,8867 & 0,9957 & 0,9872 \\
\hline 296 & 1,0000 & 0,9963 & 1,0000 & 1,0000 & 1,0000 \\
\hline 297 & 1,0000 & 1,0000 & 1,0000 & 1,0000 & 1,0000 \\
\hline 298 & 0,9912 & 0,9624 & 0,8867 & 0,9897 & 0,9985 \\
\hline 393 & 0,9963 & 0,9916 & 0,8867 & 0,9940 & 0,9544 \\
\hline 395 & 1,0000 & 1,0000 & $-0,7500$ & 0,9375 & 1,0000 \\
\hline 397 & 1,0000 & 1,0000 & $-0,7500$ & 1,0000 & 1,0000 \\
\hline 406 & 0,9918 & 0,9877 & 0,6650 & 0,9959 & 1,0000 \\
\hline 415 & 0,9898 & $-0,6910$ & $-1,0000$ & 0,9985 & $-0,9880$ \\
\hline
\end{tabular}


Tabela 3

Setores censitários segundo os diferentes potenciais de exposição às condições de risco para as infecções respiratórias agudas e composição de grupos homogêneos com base em índice final. Butantã, São Paulo, Brasil, 1998.

\begin{tabular}{|c|c|c|c|c|c|}
\hline Setor censitário & $\begin{array}{c}\text { Índice de inserção } \\
\text { social (ISS) }\end{array}$ & $\begin{array}{c}\text { Setor } \\
\text { censitário }\end{array}$ & $\begin{array}{l}\text { Índice de qualidade } \\
\text { do domicílio (IQD) }\end{array}$ & $\begin{array}{c}\text { Setor } \\
\text { censitário }\end{array}$ & $\begin{array}{c}\text { Índice } \\
\text { final }\end{array}$ \\
\hline 296 & 0,9457 & 297 & 1,0000 & 296 & 0,9635 \\
\hline 297 & 0,9347 & 296 & 0,9992 & 297 & 0,9565 \\
\hline 236 & 0,9048 & 4 & 0,9622 & 236 & 0,9232 \\
\hline 2 & 0,8985 & 5 & 0,9622 & 2 & 0,9196 \\
\hline 237 & 0,8840 & 85 & 0,9622 & 165 & 0,8931 \\
\hline 165 & 0,8619 & 18 & 0,9618 & 1 & 0,8724 \\
\hline 1 & 0,8296 & 2 & 0,9617 & 4 & 0,8542 \\
\hline 4 & 0,8002 & 3 & 0,9605 & 18 & 0,8450 \\
\hline 18 & 0,7866 & 236 & 0,9600 & 237 & 0,8133 \\
\hline 238 & 0,7383 & 6 & 0,9598 & 238 & 0,8103 \\
\hline 393 & 0,7381 & 7 & 0,9598 & 6 & 0,8103 \\
\hline 6 & 0,7355 & 1 & 0,9580 & 393 & 0,8084 \\
\hline 85 & 0,7039 & 165 & 0,9555 & 85 & 0,7900 \\
\hline 30 & 0,5613 & 238 & 0,9543 & 5 & 0,6725 \\
\hline 161 & 0,5434 & 298 & 0,9514 & 3 & 0,6658 \\
\hline 43 & 0,5343 & 19 & 0,9512 & 161 & 0,6572 \\
\hline 5 & 0,5276 & 393 & 0,9491 & 7 & 0,6470 \\
\hline 3 & 0,5184 & 161 & 0,8847 & 19 & 0,6303 \\
\hline 7 & 0,4906 & 406 & 0,8842 & 9 & 0,6140 \\
\hline 9 & 0,4820 & 9 & 0,8780 & 406 & 0,5770 \\
\hline 19 & 0,4698 & 237 & 0,6717 & 298 & 0,5741 \\
\hline 406 & 0,4234 & 397 & 0,4167 & 30 & 0,5109 \\
\hline 298 & 0,3854 & 147 & 0,4136 & 43 & 0,4936 \\
\hline 8 & $-0,0089$ & 43 & 0,4121 & 8 & 0,1299 \\
\hline 29 & $-0,0112$ & 83 & 0,4115 & 29 & 0,1277 \\
\hline 147 & $-0,0197$ & 30 & 0,4101 & 397 & 0,1255 \\
\hline 397 & $-0,0202$ & 395 & 0,4097 & 147 & 0,1248 \\
\hline 39 & $-0,0459$ & 39 & 0,4096 & 39 & 0,1059 \\
\hline 83 & $-0,0507$ & 8 & 0,4077 & 83 & 0,1034 \\
\hline 395 & $-0,0605$ & 36 & 0,4063 & 395 & 0,0962 \\
\hline 160 & $-0,4378$ & 11 & 0,4059 & 160 & $-0,1567$ \\
\hline 162 & $-0,4740$ & 31 & 0,4058 & 162 & $-0,1836$ \\
\hline 35 & $-0,5002$ & 29 & 0,4056 & 11 & $-0,1986$ \\
\hline 11 & $-0,5009$ & 160 & 0,4054 & 36 & $-0,2024$ \\
\hline 36 & $-0,5067$ & 144 & 0,3997 & 35 & $-0,2033$ \\
\hline 144 & $-0,5350$ & 162 & 0,3972 & 144 & $-0,2235$ \\
\hline 31 & $-0,5579$ & 35 & 0,3904 & 31 & $-0,2366$ \\
\hline 33 & $-0,5790$ & 33 & 0,2998 & 33 & $-0,2861$ \\
\hline 46 & $-0,7189$ & 34 & $-0,1252$ & 46 & $-0,5850$ \\
\hline 37 & $-0,7370$ & 46 & $-0,3174$ & 38 & $-0,6970$ \\
\hline 45 & $-0,7683$ & 38 & $-0,3602$ & 34 & $-0,7024$ \\
\hline 44 & $-0,7770$ & 10 & $-0,3837$ & 10 & $-0,7196$ \\
\hline 38 & $-0,8654$ & 415 & $-0,4855$ & 37 & $-0,7307$ \\
\hline 84 & $-0,8725$ & 32 & $-0,5072$ & 45 & $-0,7712$ \\
\hline 10 & $-0,8875$ & 148 & $-0,7054$ & 415 & $-0,7751$ \\
\hline 148 & $-0,8999$ & 84 & $-0,7156$ & 44 & $-0,7777$ \\
\hline 415 & $-0,9198$ & 37 & $-0,7180$ & 32 & $-0,8007$ \\
\hline 32 & $-0,9474$ & 45 & $-0,7769$ & 84 & $-0,8202$ \\
\hline 34 & $-0,9911$ & 44 & $-0,7791$ & 148 & $-0,8351$ \\
\hline
\end{tabular}




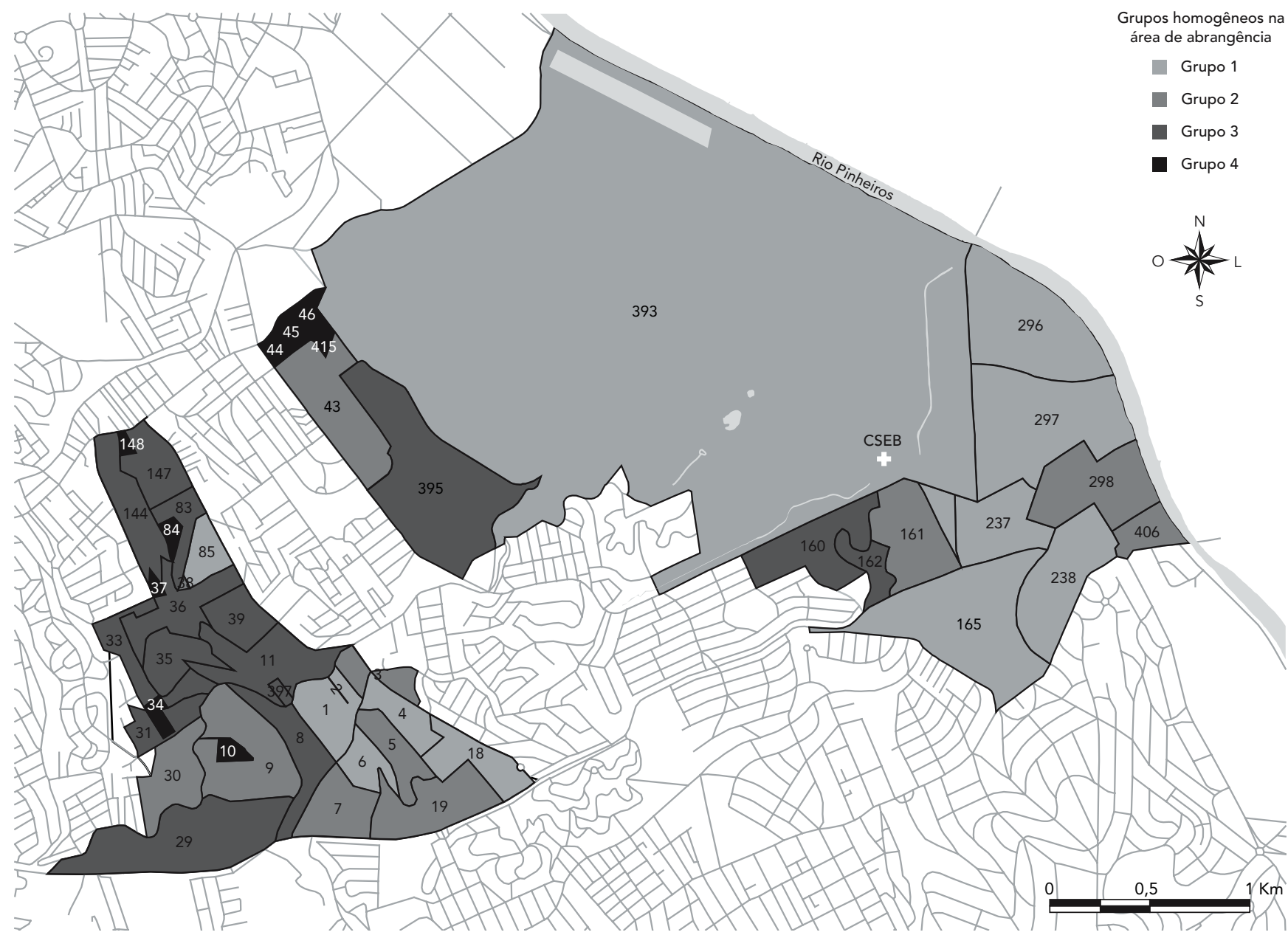

cinco anos desde 1991 até 1997, na região em questão. Para tal, foi solicitado ao Programa de Aprimoramento de Informações sobre Mortalidade no Município de São Paulo (PROAIM) as informações a respeito dessas mortes. Foram excluídas do banco de dados 121 crianças por endereços incompletos, visto não aparecia o nome da rua, número complemento e subdistrito, provavelmente relativos às famílias que ocupam o território de forma irregular e que não puderam ser localizadas. Foi possível mapear 148 óbitos de menores de cinco anos de idade na área de abrangência. Nesta etapa, foi utilizado especialmente o programa MapInfo.

A Figura 5 apresenta a distribuição dos 148 óbitos por todas as causas, de menores de cinco anos, ocorridos no período de 1993 a 1997 nas famílias residentes na área estudada.

Os coeficientes de mortalidade de crianças em diversos países evidenciam que se trata de um fenômeno relacionado à forma de organização social, constituindo, por isso, um excelente indicador de desenvolvimento social. A partir do momento em que a sociedade humana detém conhecimentos suficientes para intervir sobre os agravos capazes de provocar a morte nessa faixa etária de grande vulnerabilidade, os profissionais de saúde passam a enfrentar um problema de ordem técnica e de ordem política, uma vez que o problema tem relação direta com a distribuição e o acesso aos bens produzidos pelos diferentes segmentos da sociedade. Na realidade brasilei- 
Figura 3

Distribuição percentual dos domicílios nos grupos homogêneos.

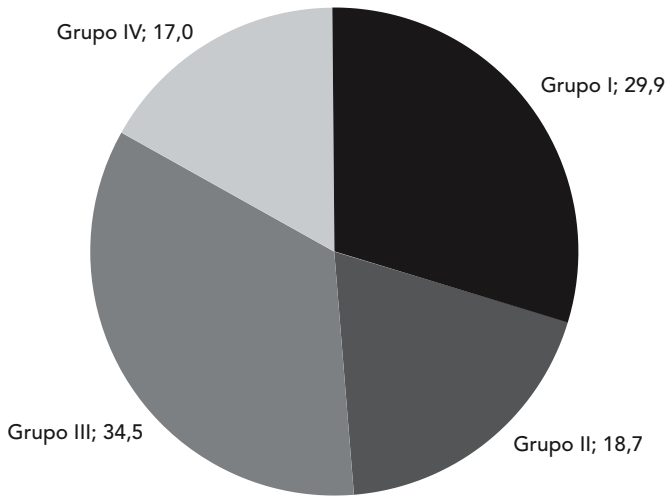

Figura 4

Distribuição percentual da população de menores de cinco anos nos grupos homogêneos.

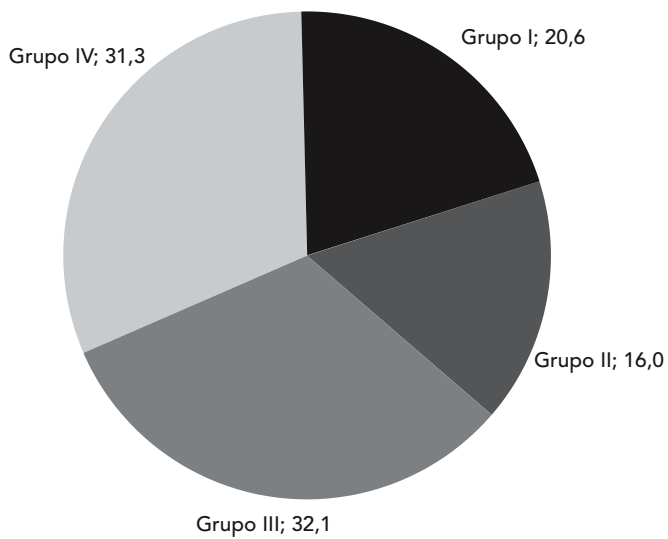

ra, o aspecto da distribuição mais eqüitativa se configura como um dos grandes problemas a ser enfrentado, já que o Brasil vem apresentando uma situação de grande desigualdade interna ao longo dos últimos trinta anos.

Segundo Cohn 29 , enquanto na década de 60 a parcela representada pelos $10 \%$ mais ricos da população apresentava uma concentração de renda 34 vezes maior do que os $10 \%$ mais pobres.
Segundo Kunst \& Mackenbach 30 , correlacionar os dados de mortalidade aos dados sócioeconômicos coletados nos censos é uma medida efetiva para mensurar desigualdades sociais e mortalidade que tem sido utilizada em vários países.

No presente estudo, a distribuição dos óbitos foi utilizada como exercício de operacionalização do mapeamento, não assumindo um caráter de estudo específico sobre o problema.

Outro aspecto que merece ser destacado é a dificuldade em se trabalhar com um evento raro numa pequena área de extensão territorial. Ainda assim, verificou-se que no decorrer no período compreendido entre os anos de 1991 a 1997, o maior número de mortes de menores de cinco anos ocorreu nos setores componentes do grupo homogêneo III, com 55 mortes $(37,2 \%)$; seguido do grupo IV, com 47 mortes $(31,8 \%)$; do grupo I, com 29 mortes (19,6\%); e finalmente do grupo II, com 17 mortes (11,5\%). Portanto, do total de 148 mortes, $69 \%$ ocorreram em famílias dos grupos III e IV, do ranking de potencial de exposição às condições de risco para as doenças respiratórias agudas. Isso mostra a necessidade de intervenções específicas no âmbito da promoção em saúde, voltadas para esses segmentos da população.

De acordo com os dados apresentados na Figura 4, referentes à distribuição da população de menores de cinco anos nos diferentes grupos homogêneos, pôde-se verificar que a ocorrência de óbitos pouco diferiu em função do número de crianças incluídas nos grupos homogêneos.

Nos grupos I e II, o percentual de óbitos ficou aquém da concentração de crianças; nos grupos III e IV, os óbitos registrados superaram um pouco a concentração de crianças nos setores envolvidos.

Tomando-se por base somente os dados relativos às mortes por pneumonia e excluindo-se a faixa etária que compreende o período neonatal, foram encontrados 16 óbitos por pneumonia, num total de 69 mortes, representando $20,3 \%$. Tais mortes ocorreram quase que exclusivamente nos grupos III e IV e, já que são amplas as possibilidades de detecção e tratamento precoce das mesmas, constituem mortes evitáveis. $\mathrm{O}$ fato por si só revela uma face das iniqüidades com as quais se pretende trabalhar.

A presente investigação contou com outra etapa de cunho qualitativo em que foram entrevistados responsáveis por crianças residentes dos diversos grupos homogêneos, identificados acerca da vivência em relação ao adoecimento por doenças respiratórias agudas. Os resultados dessa etapa serão objeto de outro artigo. 
Distribuição de óbitos por pneumonia de crianças menores de cinco anos da área de abrangência do Centro de Saúde Escola Samuel B. Pessoa. Butantã, São Paulo, Brasil, 1991-1997.

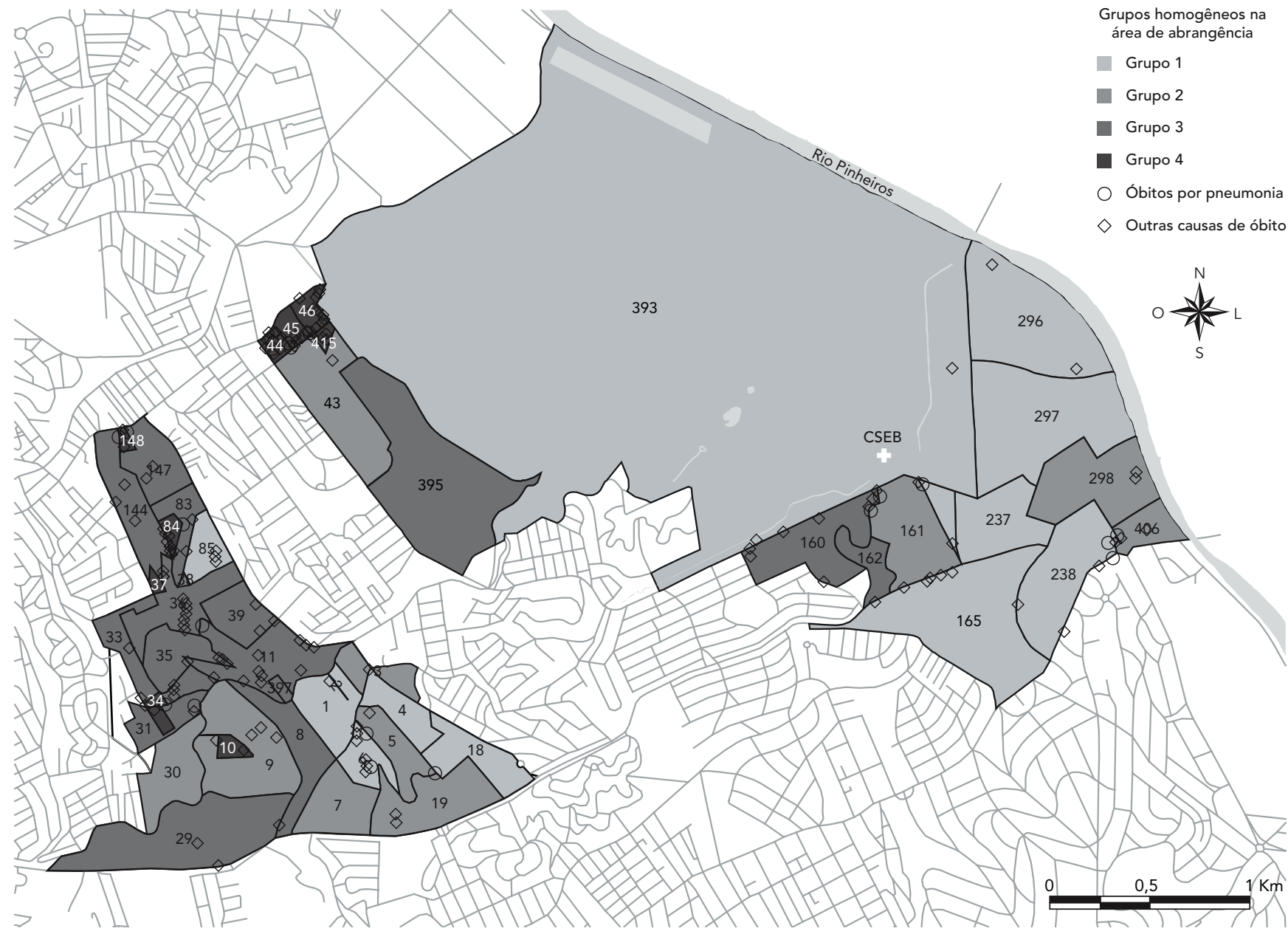

\section{Considerações finais}

A abordagem do conceito de iniqüidade é mais complexa que o de desigualdade. Por conseguinte, é importante ressaltar que o planejamento de ações centradas no princípio da eqüidade deve ampliar os poderes dos indivíduos e grupos para atuar nos processos geradores da saúde-doença. O conjunto das iniqüidades observadas no território estudado baseia-se na possibilidade de os diferentes grupos homogêneos controlarem as situações de risco.

O desafio da promoção à saúde, sobretudo nos países em que as desigualdades sociais são mais expressivas, está centrado na transforma- ção das relações excludentes, por intermédio de estratégias de trabalho intersetorial que conciliem interesses para ampliar o desenvolvimento e a garantia do bem-estar social como condição indispensável para a melhor qualidade de vida. Nessa perspectiva, a saúde deve ser entendida não como uma finalidade em si, mas sim como um meio para a garantia da qualidade de vida.

Segundo Nwokedi 31, não tem sido fácil a tarefa de promover eqüidade. Um elemento necessário, todavia não suficiente, para contemplar essa tarefa está relacionado à obtenção de dados da realidade que devem revelar a magnitude do problema e as causas das disparidades encontradas. $\mathrm{O}$ autor analisa que ainda faltam 
medidas concretas para avaliar o impacto das ações voltadas para a promoção da eqüidade, bem como para mensurar as iniqüidades por classe, sexo e níveis de saúde por área geográfica.

O presente estudo buscou apresentar uma alternativa metodológica para identificação das iniqüidades no âmbito do nível local de assistência à saúde, baseando-se em determinada área geográfica. Estudos multicêntricos têm evidenciado a influência das condições de moradia adversas na mortalidade geral 32 , fato que reforça a utilização de metodologia semelhante no reconhecimento das áreas de maior vulnerabilidade social para atuação diferenciada.

Recuperando-se os objetivos do presente estudo, pode-se concluir que os resultados encontrados possibilitaram a localização geográfica de maior e menor concentração das carências relativas às condições de vida. Os métodos utilizados permitiram, numa primeira instância, identificar os aspectos quantificáveis, permitindo a comparação entre os setores censitários constitutivos da área. Logo, puderam ser conhecidas as desigualdades sociais no âmbito da microárea pela identificação de quatro grupos homogêneos com relação ao potencial de exposição às condições de risco para as doenças respiratórias das crianças menores de cinco anos de idade.

O Brasil é um país com grandes desigualdades sociais decorrentes do modelo econômico excludente. Em tal cenário, a promoção à saúde, a exemplo de outros países que também albergam problemas estruturais semelhantes, tem à frente o desafio de transformar as relações sociais excludentes.
No contexto brasileiro, o campo da promoção à saúde é recente, ainda em expansão, contudo com enorme potencial, dadas as demandas emergentes decorrentes da descentralização preconizada pelo SUS na Constituição Federal de 1988, que favoreceram o desenvolvimento de um movimento municipalista da saúde e o fortalecimento da participação popular e o controle social da população nas questões de saúde.

O uso de indicadores compostos e a construção do "índice de potencial de exposição" às condições de risco para os agravos respiratórios permitiram a visualização geográfica das áreas de maior e menor privação no território escolhido, evidenciando grupos homogêneos prioritários para a formulação de ações específicas. Entende-se que a presente investigação poderá auxiliar a realização de outros estudos sobre morbidade dos diferentes grupos homogêneos, semelhantes à investigação realizada por Issler et al. 33 , aprimorando-se os indicadores construídos neste processo, a fim de que o método descrito possa ser reproduzido em outros contextos subsidiando o planejamento local.

Nesse sentido, destaca-se que as técnicas de geoprocessamento abriram um profícuo campo para o desenvolvimento de outras pesquisas, não somente no âmbito da saúde infantil, nem tampouco circunscrito à área da saúde, constituindo um excelente instrumento para a aproximação de diferentes setores responsáveis por políticas públicas em dada realidade. A esse respeito, ressalta-se a importância da participação de outros atores sociais no processo investigativo, o que efetivamente pode incentivar futuras articulações intersetoriais, contribuindo para envolver outros segmentos sociais no debate sobre os determinantes das condições de saúde. 


\section{Resumo}

Apresentam-se as desigualdades em saúde valendose das doenças respiratórias agudas na infância na área de abrangência de um centro de saúde na cidade de São Paulo, Brasil, para subsidiar o planejamento local de ações de promoção da saúde. O trabalho se fundamentou em estudos ecológicos que utilizam a área geográfica como unidade de análise, permitindo a comparação de indicadores de saúde e sócio-econômicos mediante dados censitários. Foram construídos indicadores de "inserção social" e de "qualidade do domicílio", gerando o "indice potencial de exposição" que reflete as condições de risco para o adoecimento por agravos respiratórios. O tratamento estatístico incluiu o agrupamento pela técnica de cluster. Foram identificados quatro grupos sociais homogêneos nas condições de risco para as doenças respiratórias agudas. Os grupos III e IV-com as piores condições sócio-econômicas - mostraram diferenciais importantes em relação aos grupos I e II. Os diferenciais de mortes por pneumonia encontrados, sugerem iniqüidades em saúde. Os resultados permitiram a localização geográfica de maior e menor concentração das carências relativas às condições de vida e a comparação entre os setores censitários para o reconhecimento de necessidades distintas, que subsidiem propostas para a articulação intersetorial.

Doenças Respiratórias; Bem-Estar da Criança; Desigualdades em Saúde

\section{Referências}

1. Organización Panamericana de la Salud/Organización Mundial de la Salud. Programa Especial de Salud Materno-Infantil y Población. Control de las infecciones respiratórias agudas: bases técnicas para las recomendaciones de la OPS/OMS. Quito: Organización Panamericana de la Salud/Organización Mundial de la Salud; 1993.

2. Sigaud CHS. Concepções e práticas maternas relacionadas à criança com pneumonia: estudo realizado no município de São Paulo [Tese de Doutorado]. São Paulo: Faculdade de Saúde Pública, Universidade de São Paulo; 2003.

3. Antuñano FJL. Epidemiologia das infecções respiratórias agudas em crianças: panorama regional. In: Benguigui Y, Antuñano FJL, Schmunis G, Yunes J, organizadores. Infecções respiratórias em crianças. Washington DC: Organização Pan-Americana da Saúde; 1998. p. 3-22. (Série HCT/AIEPI-1.P).

4. Graham NM. The epidemiology of acute respiratory infections in children and adults: a global perspective. Epidemiol Rev 1990; 12:140-78.

\section{Colaboradores}

Asseguramos que todos os autores participaram da concepção da pesquisa, bem como da análise, interpretação dos dados, de sua redação e revisão critica. Todos os autores participaram igualmente das modificações apresentadas e aprovaram a versão final.

\section{Agradecimentos}

Fundação de Amparo à Pesquisa do Estado de São Paulo (FAPESP) pelo apoio financeiro.
5. Fundo das Nações Unidas para a Infância/Instituto Brasileiro de Geografia e Estatística. Municípios brasileiros: crianças e suas condições de sobrevivência. Brasília: Fundo das Nações Unidas para a Infância; 1994.

6. Yunes J, Díaz A. Situação da saúde materno-infantil e suas tendências na América Latina e no Caribe. In: Bengui Y, Land S, Paganini JM, Yunes J, organizadores. Ações de saúde materno-infantil em nível local, segundo a cúpula mundial em favor da criança. Washington DC: Organização Pan-Americana da Saúde; 1997. p. 1-16. (Série HCT/AIEPI-4).

7. Victora CG. Fatores de risco nas IRA baixas. In: Benguigui Y, Antuñano FJL, Schmunis G, Yunes J, organizadores. Infecções respiratórias em crianças. Washington DC: Organização Pan-Americana da Saúde; 1998. p. 43-61. (Série HCT/AIEPI-1.P). 
8. Niobey FML, Duchiade MP, Vasconcelos AGG, Carvalho ML, Leal MC, Valente JG. Fatores de risco para morte por pneumonia em menores de um ano em uma região metropolitana do Sudeste do Brasil: um estudo tipo caso-controle. Rev Saúde Pública 1992; 26:229-38.

9. Fonseca W, Kirkwood BR, Victora CG, Fuchs SR, Flores JA, Misago C. Fatores de risco para pneumonia em menores de dois anos de idade em Fortaleza, Brasil: um estudo de casos e controles. In: Benguigui Y, organizador. Investigações operacionais sobre o controle das infecções respiratórias agudas (IRA). Washington DC: Organização Pan-Americana da Saúde/Organização Mundial da Saúde; 1997. p. 71-84. (Série HCT/AIEPI-2).

10. Amaral JJF, Menezes AMB, Halpern R, Victora CG, Barros FC. Prevalência e fatores de risco para infecção respiratória aguda em crianças aos seis meses de vida em Pelotas, RS. In: Benguigui Y, organizador. Investigações operacionais sobre o controle das infecções respiratórias agudas (IRA). Washington DC: Organização Pan-Americana da Saúde/Organização Mundial da Saúde; 1997. p. 85-97. (Série HCT/AIEPI-2).

11. Paganini JM, Capote Mir R. Los sistemas locales de salud: conceptos, métodos, experiencias. Washington DC: Organización Panamericana de la Salud; 1990. (Publicación Científica, 519).

12. Schraiber LB, Mendes-Gonçalves, RB. Necessidades de saúde e atenção primária. In: Schraiber LB, Nemes MIB, Mendes-Gonçalves RB, organizadores. Saúde do adulto: programas e ações na unidade básica. São Paulo: Editora Hucitec; 1996. p. 29-47.

13. Kadt E, Tasca R. Promovendo a eqüidade: um novo enfoque com base no setor da saúde. São Paulo: Editora Hucitec/Salvador: Cooperativa Italiana em Saúde; 1993.

14. Whitehead M. The concepts and principles of equity and health. Copenhagen: World Health Organization; 1990.

15. Santos M. Técnica, espaço, tempo: globalização e meio técnico-científico informacional. São Paulo: Editora Hucitec; 1994.

16. Unglert CVS. Territorialização em sistemas de saúde. In: Mendes EV, organizador. Distrito sanitário: o processo social de mudança das práticas sanitárias no Sistema Único de Saúde. São Paulo: Editora Hucitec/Rio de Janeiro: ABRASCO; 1995. p. 221-35.

17. Ammann SB. Ideologia do desenvolvimento de comunidade no Brasil. São Paulo: Cortez Editora; 1985.

18. Teixeira CF. Planejamento e programação situacional em distritos sanitários: metodologia e organização. In: Mendes EV, organizador. Distrito sanitário: o processo social de mudança das práticas sanitárias no Sistema Único de Saúde. São Paulo: Editora Hucitec/Rio de Janeiro: ABRASCO; 1995. p. 237-65.

19. Paim JS. Abordagens teórico-conceituais em estudos de condições de vida e saúde: notas para reflexão e ação. In: Barata RB, organizador. Condições de vida e situação de saúde. Rio de Janeiro: ABRASCO; 1997. p. 7-30.
20. Sposati A, organizadora. Mapa da exclusão/inclusão social da cidade de São Paulo. São Paulo: EDUC; 1996.

21. Instituto Brasileiro de Geografia e Estatística. Censo demográfico de 1991. Rio de Janeiro: Instituto Brasileiro de Geografia e Estatística; 1991.

22. Borrel C. Métodos utilizados no estudo das desigualdades sociais em saúde. In: Barata RB, organizador. Condições de vida e situação de saúde. Rio de Janeiro: ABRASCO; 1997. p. 167-95.

23. Castellanos PL. Epidemiologia, saúde pública, situação de saúde e condições de vida: considerações conceituais. In: Barata RB, organizador. Condições de vida e situação de saúde. Rio de Janeiro: ABRASCO; 1997. p. 31-75.

24. Akerman M, Campanário P, Maia PB. Saúde e meio ambiente: análise de diferenciais intra-urbanos, Município de São Paulo, Brasil. Rev Saúde Pública 1996; 30:373-82.

25. Barata RCB, Waldman EA, Moraes JC, Guibu IA, Rossov T, Takimoto S. Gastroenterites e infecções respiratórias agudas em crianças menores de 5 anos em área da região Sudeste do Brasil, 19861987. I. Infecções respiratórias agudas. Rev Saúde Pública 1996; 30:553-63.

26. Monteiro CA, Benício MHD’A. Estudo das condições de saúde das crianças do Município de São Paulo, SP (Brasil), 1984-1985. VI. Doença respiratória. Rev Saúde Pública 1987; 21:380-6.

27. Cardoso MR. Indoor environmental risk factors for lower respiratory diseases in young children in São Paulo, Brazil [Tese de Doutorado]. Londres: University of London; 1997.

28. Fundação Sistema Estadual de Análise de Dados. Pesquisa de condições de vida na Região Metropolitana de São Paulo. São Paulo: Fundação Sistema Estadual de Análise de Dados; 1992.

29. Cohn A. Desenvolvimento social e impactos na saúde In: Barata RB, organizador. Condições de vida e situação de saúde. Rio de Janeiro: ABRASCO; 1997. p. 77-93.

30. Kunst AE, Mackenbach JP. Measuring socioeconomic inequalities in health. Copenhagen: World Health Organization; 1995.

31. Nwokedi J. Equity in health. Promotion \& Education 1998; 5:13-7.

32. van Lenth FJ, Borrell LN, Costa G, Diez Roux AV, Kauppinen TM, Marinacci C, et al. Neighbourhood unemployment and all cause mortality: a comparison of six countries. J Epidemiol Community Health 2005; 59:231-7.

33. Issler RMS, Giugliani ERJ, Kreutz GT, Meneses CF, Justo EB, Kreutz VM, et al. Poverty levels and children's health status: study risck factors in an urban population of low socioeconomic level. Rev Saúde Pública 1996; 30:506-11.

Recebido em 07/Abr/2006

Versão final reapresentada em 12/Fev/2007

Aprovado em 19/Abr/2007 